\title{
A rotating molecular jet in Orion
}

\author{
L. A. Zapata, J. Schmid-Burgk, D. Muders, P. Schilke, K. Menten, and R. Guesten
}

\author{
Max-Planck-Institut für Radioastronomie, Auf dem Hügel 69, 53121 Bonn, Germany \\ e-mail: lzapata@mpifr-bonn.mpg.de
}

Received 22 May 2008 / Accepted 23 October 2009

\begin{abstract}
We present $\mathrm{CO}(2-1),{ }^{13} \mathrm{CO}(2-1), \mathrm{CO}(6-5), \mathrm{CO}(7-6)$, and $\mathrm{SO}\left(6_{5}-5_{4}\right)$ line observations made with the IRAM $30 \mathrm{~m}$ and Atacama Pathfinder Experiment (APEX) radiotelescopes and the Submillimeter Array (SMA) toward the highly collimated $\left(11^{\circ}\right)$ and extended $\left(\sim 2^{\prime}\right)$ southwest lobe of the bipolar outflow Ori-S6 located in the Orion South region. We report for all these lines, the detection of velocity asymmetries about the flow axis with velocity differences roughly on the order of $1 \mathrm{~km} \mathrm{~s}^{-1}$ over distances of about $5000 \mathrm{AU}$, $4 \mathrm{~km} \mathrm{~s}^{-1}$ over distances of about $2000 \mathrm{AU}$, and close to the source of between 7 and $11 \mathrm{~km} \mathrm{~s}^{-1}$ over smaller scales of about 1000 AU. The redshifted gas velocities are located to the southeast of the outflow's axis, the blueshifted ones to the northwest. We interpret these velocity differences as a signature of rotation, but also discuss some alternatives which we recognize as unlikely in view of the asymmetries' large downstream continuation. In particular, any straightforward interpretation by an ambient velocity gradient does not seem viable. This rotation across the Ori-S6 outflow is observed out to (projected) distances beyond $2.5 \times 10^{4}$ AU from the flow's presumed origin. Comparison of our large-scale (single dish) and small-scale (SMA) observations suggests the rotational velocity to decline not faster than $1 / R$ with distance $R$ from the axis; in the innermost few arcsecs an increase of rotational velocity with $R$ is even indicated. The magnetic field lines threading the inner rotating CO shell may well be anchored in a disk of a radius of $\sim 50 \mathrm{AU}$; the field lines further out need a more extended rotating base. Our high angular resolution SMA observations also suggest this outflow to be energized by the compact millimeter radio source 139-409, a circumbinary flattened ring that is located in a small cluster of very young stars associated with the extended and bright source FIR4.
\end{abstract}

Key words. ISM: jets and outflows - techniques: high angular resolution - binaries: general - stars: pre-main sequence ISM: molecules - radio lines: ISM

\section{Introduction}

Protostellar jets have the essential task of removing angular momentum from the cores of pre-/protostellar clouds in order for these to contract into new stellar objects. It is believed that $\mathrm{T}$ Tauri winds and protostellar jets are driven magnetocentrifugally from keplerian accretion disks close to the central stars (for a review see Königl \& Pudritz 2000; Shu et al. 2000; Pudritz et al. 2007; Shang et al. 2007). In these models the magnetic fields that are anchored in the accretion disk-star system are responsible for accelerating the jets' material from the accretion disk. The material ejected from the disk therefore possesses angular momentum and, if not completely free to move away from the jet immediately, will thus show a toroidal velocity component, i.e. rotation about the jet axis.

In recent years a number of observations at optical wavelengths, using high spectral and angular resolution toward the launching zones of young jets from $\mathrm{T}$ Tauri stars, have attempted to identify velocity asymmetries that might be interpreted as signatures of jet rotation. These observations include Bacciotti et al. (2002), Coffey et al. (2004), Woitas et al. (2005), and Coffey et al. (2007) who detected systematic velocity shifts (of order 5 to $25 \mathrm{~km} \mathrm{~s}^{-1}$ ) across the jet's axis within the first 100-200 AU from the star, toward six T Tauri stars, e.g. DG tau, RW Aur, CW Tau, and Th 28. Furthermore, observations at infrared wavelengths have also revealed such velocity jumps in the launching zones of the Herbig-Haro objects HH 26 and HH 72 (Chrysostomou et al. 2008). All these authors have interpreted the velocity shifts as a signature of jet rotation produced by a magneto centrifugal wind.

The first tentative evidence of such an outflow rotation was presented by Davis et al. (2000) at infrared wavelengths. They reported velocity shifts of a few $\mathrm{km} \mathrm{s}^{-1}$ across the $\mathrm{HH} 212$ jet at distances of about $10^{4} \mathrm{AU}$ from the ejecting object, using spectral line observations of the molecule $\mathrm{H}_{2}$.

At millimeter wavelengths there have likewise been attempts to find such signatures near the base of strong molecular outflows (HH 30: Pety et al. 2006; HH212: Codella et al. 2007; Lee et al. 2008; HH 211: Lee et al. 2007; CB 26: Launhardt et al. 2009). However, there seems to be evidence of rotation only in the CB 26, the HH212, and the HH 211 outflows.

Recent studies have proposed alternative explanations for observed velocity asymmetries in the jets. Soker (2005) remarked that the interaction of the jet with a twisted-tilted (warped) accretion disk can lead to the observed asymmetry in the jet's line-of-sight velocity profile, and thus the magneto centrifugal wind acceleration model is not required to explain such velocity jumps at the base of the optical and infrared jets. Cerqueira et al. (2006) proposed that a precessing jet whose ejection velocity changes periodically with a period equal to the precession period could also reproduce the line profiles of the jets.

Below, we address the issue of outflow rotation by combining new interferometer and single-dish observations of the Ori-S6 molecular outflow (Schmid-Burgk et al. 1990), which is located in the southernmost part of the very active high- and intermediate-mass star forming region Orion South. 
Single-dish observations suggested this outflow to originate near the millimeter sources CS3/FIR4 some $100^{\prime \prime}$ to the south of the $\mathrm{BN} / \mathrm{KL}$ object. Unfortunately the immediate vicinity of CS3/FIR4 contains at least two more molecular outflows (Zapata et al. 2005, 2007), both almost perpendicular to Ori-S6, so that unique differentiation between the several emitting structures becomes only possible for single telescopes beyond some $20^{\prime \prime}$ from the presumed region of origin. Interferometry however has permitted Zapata et al. (2004a,b) to propose the source of this outflow to be the elongated radio object 134-411 (possibly a compact thermal jet) that is located close to the position of CS3/FIR4, the position angle of its major axis being consistent with that of the outflow.

The redshifted lobe of Ori-S6 is highly collimated $\left(11^{\circ}\right)$, of relatively low radial velocities (up to 15 to $20 \mathrm{~km} \mathrm{~s}^{-1}$ relative to ambient) and extended over $\sim 2^{\prime}$ in a direction of PA around $210^{\circ}$. That lobe is much better defined and stronger than its blueshifted counterpart because it is interacting directly with the molecular cloud, while the latter points in direction of the HII region and is likely destroyed by it. Below, we will deal with the redshifted lobe only.

Zapata et al. (2006) suggested that this outflow could be associated with a collimated $\mathrm{SiO}$ jet that they had detected in their SMA observations and that is emanating from the CS3/FIR4 region with almost the same orientation. On larger scales and at optical wavelengths, Henney et al. (2007); O'Dell et al. (2008) discussed the possibility that the redshifted lobe of the Ori-S6 outflow is associated with a strong shock ("Southwest Shock"), some 400" downstream, that is imaged in the Fig. 6 of Henney et al. (2007).

$\mathrm{CO}$ position-velocity diagrams along the red lobe reveal a two-step structure over a distance of about $120^{\prime \prime}$ from the source region. Over the inner $60^{\prime \prime}$ there is a linear buildup of outflow velocities, from ambient (around 7 to $8 \mathrm{~km} \mathrm{~s}^{-1}$ ) to maximum values near $25 \mathrm{~km} \mathrm{~s}^{-1}$, which then terminates abruptly. At the same point the ambient velocities as seen in $\mathrm{CO}$, which closer to CS3/FIR 4 cover a rather wide range of between 7 and $9.5 \mathrm{~km} \mathrm{~s}^{-1}$, acquire an additional component, of around $10 \mathrm{~km} \mathrm{~s}^{-1}$, which further downstream becomes the dominant velocity. At this point a second, even faster linear buildup of radial velocity, again starting from ambient values, sets in which follows exactly the same direction as the first one. Quite likely the break in outflow velocity around the $60^{\prime \prime}$ point is related to the change in ambient conditions.

In the present study we will concentrate on the inner $60^{\prime \prime}$ section. Already early on Muders \& Schmid-Burgk (1992) had noted this section, as seen in $\mathrm{C}^{18} \mathrm{O}(2-1)$, to be enveloped by a somewhat symmetrical cylindrical structure that seemed to undergo rotation about the outflow axis.

Throughout the paper, we have divided the outflow into three main components: (1) on small scales ( 1000 AU), the jet (concentrated in bullets); (2) on large scales ( 5000 AU), the shell, both observed with the SMA; and (3) on very large scales $\left(\sim 10^{4} \mathrm{AU}\right)$, the envelope, observed with single dish telescopes.

We present $\mathrm{CO}(2-1),{ }^{13} \mathrm{CO}(2-1), \mathrm{CO}(6-5), \mathrm{CO}(7-6)$, and $\mathrm{SO}\left(6_{5}-5_{4}\right)$ line observations made with both the IRAM $30 \mathrm{~m}$ and APEX telescopes as well as the submillimeter array toward the redshifted lobe of Ori-S6. We report the detection at all three observatories of velocity jumps across the flow axis and interpret these as a signature of rotation. We discuss some alternative suggestions to explain these velocity asymmetries. Finally, the SMA observations suggest the source of the extended collimated outflow to be the millimeter continuum source 139-409.

\section{Observations}

\subsection{Submillimeter Array}

\subsection{1. $\mathrm{SO}\left(6_{5}-5_{4}\right)$ high resolution}

Observations were made with the Submillimeter Array (SMA) ${ }^{1}$ during 2004 September 3. The SMA was in its extended configuration, which includes 21 independent baselines ranging in projected length from 16 to $180 \mathrm{~m}$. The phase reference center of the observations was RA $=05^{\mathrm{h}} 35^{\mathrm{m}} 14^{\mathrm{s}}$, dec $=$ $-05^{\circ} 24^{\prime} 00^{\prime \prime}$ (J2000.0). The size of the primary beam response at this frequency is $50^{\prime \prime}$. The receivers were tuned to a frequency of $230.534 \mathrm{GHz}$ in the upper sideband (USB), while the lower sideband (LSB) was centered on $220.534 \mathrm{GHz}$.

The $\mathrm{SO}\left(6_{5}-5_{4}\right)$ transition was detected in the LSB at a frequency of $219.9 \mathrm{GHz}$. The full bandwidth of the SMA correlator is $4 \mathrm{GHz}$ ( $2 \mathrm{GHz}$ in each band). The SMA digital correlator was configured in 24 spectral windows ("chunk") of $104 \mathrm{MHz}$ each, with 32 channels distributed over each spectral window, providing a resolution of $3.25 \mathrm{MHz}\left(4.29 \mathrm{~km} \mathrm{~s}^{-1}\right)$ per channel.

The zenith opacity $\left(\tau_{230 \mathrm{GHz}}\right)$, measured with the NRAO tipping radiometer located at the Caltech Submillimeter Observatory, was $\sim 0.09$, indicating very good weather conditions during the experiment. Observations of Callisto provided the absolute scale for the flux density calibration. Phase and amplitude calibrators were the quasars 0423-013 and 3C 120, with measured flux densities of $2.30 \pm 0.06$ and $0.50 \pm 0.03 \mathrm{Jy}$, respectively. The absolute flux density calibration uncertainty is estimated to be $20 \%$, based on SMA monitoring of quasars. Further technical descriptions of the SMA and its calibration schemes can be found in Ho et al. (2004).

The data were calibrated using the IDL superset MIR, originally developed for the Owens Valley Radio Observatory (Scoville et al. 1993) and adapted for the SMA ${ }^{2}$. The calibrated data were imaged and analyzed in the standard manner using the MIRIAD and AIPS packages. We used the ROBUST parameter set to 0 for an optimal compromise between sensitivity and angular resolution. The line image rms-noise was $20 \mathrm{mJy}$ beam $^{-1}$ for each channel at an angular resolution of $11^{\prime \prime} 13 \times 0.93$ with a PA $=-73^{\circ}$.

\subsection{2. ${ }^{12} \mathrm{CO}(2-1)$ and $\mathrm{SO}\left(6_{5}-5_{4}\right)$ low resolution}

Observations were made during December 2007 and February 2009. The SMA was in its compact configuration, which includes 28 independent baselines ranging in projected length from 8 to $57 \mathrm{~m}$. The receivers were tuned to a frequency of $230.534 \mathrm{GHz}$ in the upper sideband (USB), while the lower sideband (LSB) was centered at $220.534 \mathrm{GHz}$. The observations were made in mosacing mode using the half-power point spacing between field centers and thus covering a total area of about $2^{\prime} \times 2^{\prime}$ in Orion South that contains the outflow reported by Schmid-Burgk et al. (1990).

The full bandwidth of the SMA correlator is $4 \mathrm{GHz}$. The SMA digital correlator was configured in 24 spectral windows of $104 \mathrm{MHz}$ each, with 128 channels distributed over each spectral window, providing a resolution of $0.812 \mathrm{MHz}$ $\left(1.05 \mathrm{~km} \mathrm{~s}^{-1}\right)$ per channel.

\footnotetext{
1 The Submillimeter Array is a joint project between the Smithsonian Astrophysical Observatory and the Academia Sinica Institute of Astronomy and Astrophysics, and is funded by the Smithsonian Institution and the Academia Sinica.

2 The MIR cookbook by C. Qi can be found at http://cfa-www . harvard. edu/ cqi/mircook.html
} 
The $\mathrm{SO}\left(6_{5}-5_{4}\right)$ and ${ }^{12} \mathrm{CO}(2-1)$ transitions were detected in the LSB and USB at a frequency of about $219.9 \mathrm{GHz}$ and $230.5 \mathrm{GHz}$, respectively.

The zenith opacity $\left(\tau_{230 \mathrm{GHz}}\right)$, was $\sim 0.1-0.3$, indicating reasonable weather conditions. Observations of Uranus provided the absolute scale for the flux density calibration. Phase and amplitude calibrators were the quasars $0530+135$ and $0541-056$.

The line image rms-noise was $150 \mathrm{mJy}^{\text {beam }}{ }^{-1}$ for each channel at an angular resolution of 3'. $44 \times 2$ '.93 with a PA $=$ $-4.9^{\circ}$. For the $\mathrm{SO}\left(6_{5}-5_{4}\right)$ we tapered our angular resolution at 6 6. $^{\prime} 027 \times 5^{\prime \prime} .131$ with a $\mathrm{PA}=-7.7^{\circ}$.

\subsection{Single-dish observations}

\subsubsection{IRAM $30 \mathrm{~m}$}

Initial $\mathrm{CO}$ and ${ }^{13} \mathrm{CO}(2-1)$ observations were performed in February 1991 at the IRAM 30 m telescope, covering a $70^{\prime \prime} \times$ $80^{\prime \prime}$ area centered $10^{\prime \prime}$ north of the presumed source of the outflow. The beam size at $220 \mathrm{GHz}$ was $13^{\prime \prime}$, the main beam efficiency 0.46 . We used position switching with the reference fixed at $30^{\prime}$ west of the grid center and chose a velocity resolution of $0.14 \mathrm{~km} \mathrm{~s}^{-1}$. Observed points were $6^{\prime \prime}$ apart, and the observation time per point was $80 \mathrm{~s}$.

These measurements were extended and complemented by ${ }^{13} \mathrm{CO}(2-1)$ OTF observations on February 2 and 4, 2008, with a total observing time per night of $45 \mathrm{~min}$. Now the center of the $100^{\prime \prime} \times 72^{\prime \prime}$ grid was placed $36^{\prime \prime}$ downstream from the presumed source and the grid rotated to align with the outflow. We co-added the OTF dumps every 6" along scans 6" apart. Velocity resolution was $0.05 \mathrm{~km} \mathrm{~s}^{-1}$. Both frequency switching (throw $15 \mathrm{MHz}$ ) and position switching were used.

\subsubsection{APEX}

Some preliminary $\mathrm{CO}(6-5)$ and (7-6) measurements were performed in October 2007 with the $12 \mathrm{~m}$ Atacama Pathfinder Experiment (APEX) telescope located on Chajnantor in Chile. The same lines were simultaneously reobserved in depth on September 21 and 22, 2008 with the $2 \times 7$-element $\mathrm{CHAMP}^{+}$ array, covering a $120^{\prime \prime} \times 120^{\prime \prime}$ field around the origin of OriS6. Zenith opacity at $5100 \mathrm{~m}$ altitude was $\sim 0.35$ at $690 \mathrm{GHz}$ and 0.42 at $806 \mathrm{GHz}$. At $690 \mathrm{GHz}$ the APEX beam is 9", at $806 \mathrm{GHz} 7.5^{\prime \prime}$. Spectral resolution was chosen to be $0.73 \mathrm{MHz}$, corresponding to 0.32 resp. $0.27 \mathrm{~km} \mathrm{~s}^{-1}$ per channel, in a way that the 2048 channels per band resulted in a total velocity coverage of 650 resp. $550 \mathrm{~km} \mathrm{~s}^{-1}$ per band, sufficient to detect the highest-velocity bullets possibly present in this very active starforming region Orion South.

The single-dish measurements were reduced with the standard CLASS software of the Gildas package ${ }^{3}$, with only firstorder baselines subtracted from the data.

\section{Results}

\subsection{The jet}

In Figs. 1, 2 and 12, we show the $\mathrm{SO}\left(6_{5}-5_{4}\right)$ redshifted emission of the innermost part of the Ori-S6 outflow as mapped with the SMA. The emission is here integrated over velocitiesm of between +8 and $+25 \mathrm{~km} \mathrm{~s}^{-1}$. Our SO observations detected only the innermost part of the outflow because of the small primary

${ }^{3}$ http://wWw.iram. fr/IRAMFR/GILDAS beam size response of the SMA and because the beam was centered to the north of the millimeter source 139-409. In Fig. 2, we also mark the primary beam of the SMA. The molecular emission is well resolved and shows a collimated jet with clumpy morphology that is being ejected from that source. The resolved spatial size of each molecular gas bullet or clump is about 1000 to $2000 \mathrm{AU}$.

Position-velocity diagrams along directions perpendicular to the SO jet (i.e. along PA $135^{\circ}$ ) are presented in Fig. 3 for four molecular gas bullets (A-D, see Fig. 2). The bullet named E, seems not to be real because it falls far outside the primary beam response of the SMA. Three of these bullets (B-D) again show velocity jumps across the symmetry axis, in the same way as those observed on large scales (IRAM 30 m, APEX, and SMA) but with larger velocity excursions ( 7 to $11 \mathrm{~km} \mathrm{~s}^{-1}$ ) over scales of $\sim 1000 \mathrm{AU}$, see Table 1, where we give the parameters of the Gaussian least square fits to the profiles. The kinematics of the molecular gas in the bullets seems consistent with a rigid body law where the velocity is proportional to the distance from the rotation axis. This feature seems least evident in the innermost bullets, perhaps because of our poor spectral resolution $\left(4 \mathrm{~km} \mathrm{~s}^{-1}\right)$ as well as their very compact size of around one SMA beam $\left(\sim 1^{\prime \prime}\right)$. Note that the molecular bullets appear to increase their radial velocities with the distance from the ejecting object (Table 1). A similar increase had already been discovered on the much larger $30 \mathrm{~m}$ scale out to $70^{\prime \prime}$ from the source in the CO flow (Schmid-Burgk et al. 1990).

Zapata et al. (2007) reported the millimeter source 139-409 to be a circumbinary molecular ring of a size of a few hundred astronomical units $(294 \mathrm{AU} \pm 14 \mathrm{AU} \times 207 \mathrm{AU} \pm 18 \mathrm{AU})$ that is produced by two intermediate-mass stars with very compact circumstellar disks of sizes and separations of less than 50 AU. The circumbinary disk is seen almost edge-on with PA $=87^{\circ}$. The redshifted molecular gas is located toward the west, the blueshifted one toward the east. Note that the sense of rotation of the molecular material in this circumbinary disk is opposite to that found in the jet.

\subsection{The shell}

Figure 1 shows a map of the high velocity $\mathrm{CO}(2-1)$ emission from the Ori-S6 redshifted lobe intensity integrated from +12 to $+23 \mathrm{~km} \mathrm{~s}^{-1}$, as obtained at the IRAM $30 \mathrm{~m}$ telescope (Schmid-Burgk et al. 1990), overlaid with both our SMA total integrated intensity $\mathrm{SO}\left(6_{5}-5_{4}\right)$ and $\mathrm{CO}(2-1)$ maps. In Fig. 13 a channel map of the SMA $\mathrm{CO}(2-1)$ emission is also shown. In Fig. 1. one can see the SO emission to trace the inner, very collimated jet ejected along a PA of $45^{\circ}$ from the source 139-409 that is located in a small cluster of young stars associated with the extended and bright source FIR4 (Zapata et al. 2006, 2007). The CO emission on the other hand delineates the more extended parts of the outflow, with its major axis bending towards a PA of some $30^{\circ}$ within a small distance from the source. The small difference between both position angles can be explained by the outflow undergoing a deflection, possibly due to the high density $\left(\sim 10^{6} \mathrm{~cm}^{3}\right)$ molecular cloud located behind the Orion Nebula as had already been suggested for other outflows populating this region (Zapata et al. 2006; Henney et al. 2007). A process for the deflection of an outflow has been proposed and modeled by Cantó \& Raga (1996). In Fig. 4, we show the continuation of the SO outflow through the the same positions of the $\mathrm{CO}(2-1)$ shell, confirming that both sections are part of the same molecular outflow. 


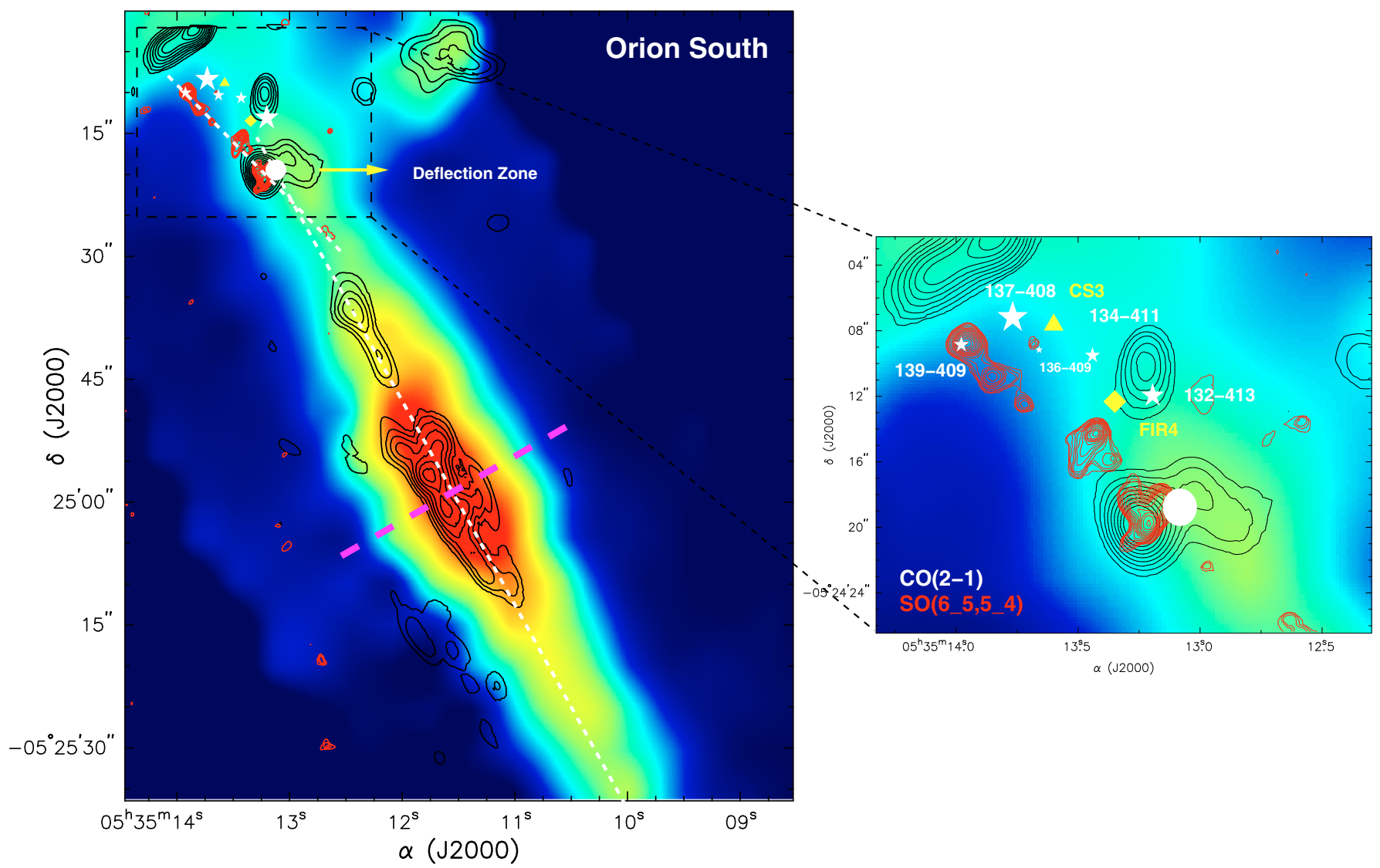

Fig. 1. IRAM $\mathrm{CO}(2-1)$ integrated intensity map of the Ori-S6 collimated molecular outflow (color image) overlaid with the SMA integrated intensity of the $\mathrm{CO}(2-1)$ (black contours) and $\mathrm{SO}\left(6_{5}-5_{4}\right)$ (red contours) images. For $\mathrm{CO}$ the contours are 4, 6, 8, 10, 12, 14, 16, 18, and 20 times $1.1 \mathrm{Jy} \mathrm{beam}^{-1} \mathrm{~km} \mathrm{~s}^{-1}$, the rms-noise of the image, while for SO the contours are 4-20 times $0.7 \mathrm{Jy} \mathrm{beam}^{-1} \mathrm{~km} \mathrm{~s}^{-1}$, the rms-noise of the image. The molecular emission in the SO map is integrated over velocities between +8 and $+25 \mathrm{~km} \mathrm{~s}^{-1}$, for the SMA CO(2-1) map from +11 to $+25 \mathrm{~km} \mathrm{~s}^{-1}$ and for the IRAM $30 \mathrm{~m} \mathrm{CO}(2-1)$ map from +12 to $+23 \mathrm{~km} \mathrm{~s}^{-1}$. The yellow diamond and triangle denote the position of the source FIR 4 (Mezger et al. 1990) and the millimeter source CS 3 (Mundy et al. 1986), respectively. The white stars mark the position of the compact millimeter sources reported by Zapata et al. (2005). Note the $\mathrm{SO}\left(6_{5}-5_{4}\right)$ maps did not cover the same large areas as the $\mathrm{CO}(2-1)$ maps from IRAM $30 \mathrm{~m}$ and SMA. The dashed white lines mark approximately the axis of the outflow before and after deflection. The white dot marks the point O. The pink line marks the position where the intensity cuts (shown in Fig. 8) were made.

Table 1. Parameters of the $\mathrm{SO}\left(6_{5}-5_{4}\right)$ line from the molecular gas bullets.

\begin{tabular}{lccc}
\hline \hline & $\begin{array}{c}\text { Peak } \\
\text { flux } \\
\text { bullet } \\
{\left[\mathrm{Jy} \mathrm{Beam}^{-1}\right]}\end{array}$ & $\begin{array}{c}\text { Half maximum } \\
\text { velocity width } \\
{\left[\mathrm{km} \mathrm{s}^{-1}\right]}\end{array}$ & $\begin{array}{c}\text { LSR radial } \\
\text { velocity } \\
{\left[\mathrm{km} \mathrm{s}^{-1}\right]}\end{array}$ \\
\hline A & $0.18 \pm 0.02$ & $11.0 \pm 2.0$ & $9.6 \pm 0.7$ \\
B & $0.28 \pm 0.02$ & $10.0 \pm 1.0$ & $12.4 \pm 0.4$ \\
C & $0.56 \pm 0.02$ & $7.6 \pm 0.4$ & $12.1 \pm 0.2$ \\
D & $0.34 \pm 0.02$ & $10.5 \pm 0.9$ & $15.0 \pm 0.4$ \\
\hline
\end{tabular}

Figure 5 presents an overlay of two $\mathrm{CO}(2-1)$ emission intervals of the south-western lobe of the Ori-S6 outflow made with the SMA, one integrated over the velocities from 14 to $16 \mathrm{~km} \mathrm{~s}^{-1}$ (blue), the other from 18 to $20 \mathrm{~km} \mathrm{~s}^{-1}$ (red). It clearly shows a velocity "jump" across the outflow, with the redshifted gas velocities located toward the south-east, the blueshifted ones toward the north-west. Note that the blue- and red-shifted velocities here are given with respect to the outflow's mean velocity at a given distance from the center. With a distance of $415 \mathrm{pc}$ to the Orion Nebula (Menten et al. 2007), the separation between these two components is about $2 \times 10^{3} \mathrm{AU}$.
The position-velocity diagram of Fig. 6, taken along the black line across the outflow in Fig. 5, displays the decrease of radial velocity in the rotating shell with distance from the flow axis. The largest radial velocity $\left(22 \mathrm{~km} \mathrm{~s}^{-1}\right)$ at this distance from the source is found right on the outflow axis, from which the velocity decreases outwards at different rates on either side. At distances of $\pm 3^{\prime \prime}$ it has diminished by almost $3 \mathrm{~km} \mathrm{~s}^{-1}$ (i.e. the shell sector rotating away from the observer) resp. $7 \mathrm{~km} \mathrm{~s}^{-1}$ (rotating towards the observer). We interpret this difference as evidence for a superposition of rotation about the axis onto the outflow's general radial velocity field. The large optical depths of the tangential line of sight through the narrow shell plus the narrow width of the rotation shell itself cause the interferometer to only see the tangential shell zones while largely overlooking the weaker, extended material closer to the axis. What is visible of this material, i.e. the bridge between the $22 \mathrm{~km} \mathrm{~s}^{-1}$ on-axis component and the $19 \mathrm{~km} \mathrm{~s}^{-1}$ shell signal (the one rotating away) has no correspondence on the other side of the axis because the emission is spread out there over the much larger velocity interval of $7 \mathrm{~km} \mathrm{~s}^{-1}$ instead of $3 \mathrm{~km} \mathrm{~s}^{-1}$. Note that the shell signals both seem to have a long structure; however, their major axes do not show the same magnitude of inclination towards the outflow axis (the short white lines mark angles of $\pm 60^{\circ}$ to the vertical of the diagram). In fact the redshifted component 
L. A. Zapata et al.: A rotating molecular jet in Orion

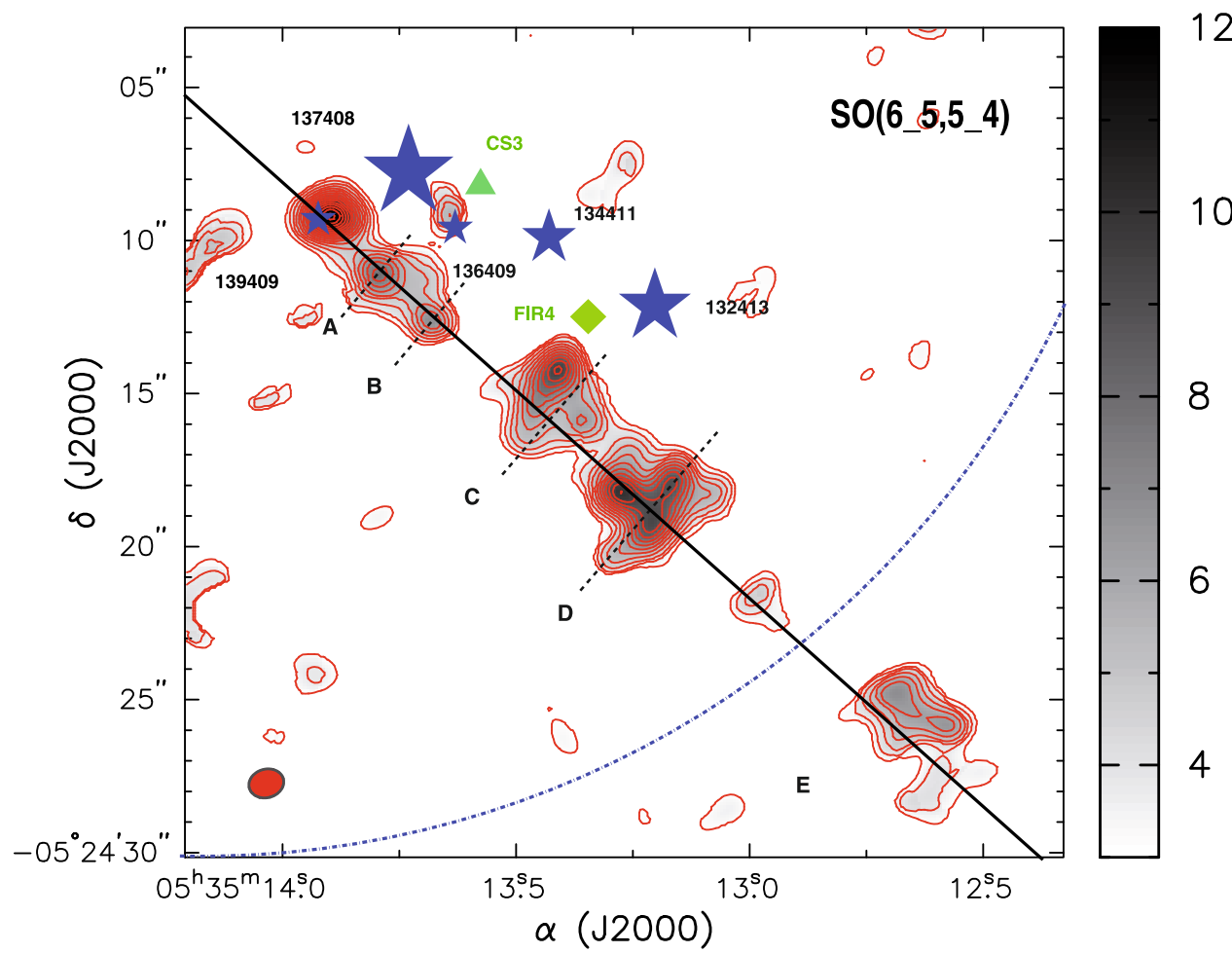

Fig. 2. SMA $\mathrm{SO}\left(6_{5}-5_{4}\right)$ integrated intensity map of the redshifted component of the Ori-S6 outflow. The contours are -3 , 3-20 times $0.7 \mathrm{Jy} \mathrm{beam}^{-1} \mathrm{~km} \mathrm{~s}^{-1}$, the rms-noise of the image. The scale bar indicates the integrated molecular emission in units of $1 \times 10^{3} \mathrm{Jy} \mathrm{beam}^{-1} \mathrm{~km} \mathrm{~s}^{-1}$. The synthesized beam is shown in the bottom left corner of the image and has a size of $1.13^{\prime \prime} \times 0.95^{\prime \prime}$ with a PA of $-73^{\circ}$. The molecular emission in the SO map is integrated over velocities between +7 and $+25 \mathrm{~km} \mathrm{~s}^{-1}$. The transversal dashed lines across the outflow represent the position of the position-velocity cuts shown in Fig. 3. The green rhombus and triangle denote the positions of the source FIR 4 (Mezger et al. 1990) and the millimeter source CS 3 (Mundy et al. 1986), respectively. The blue stars mark the position of the millimeter compact sources reported by Zapata et al. (2005). The continuous line traces approximately the symmetry axis of the outflow. The blue arc shows the position and size of the SMA primary beam. The feature $\mathrm{E}$ seems to be not real because it is outside our primary beam.
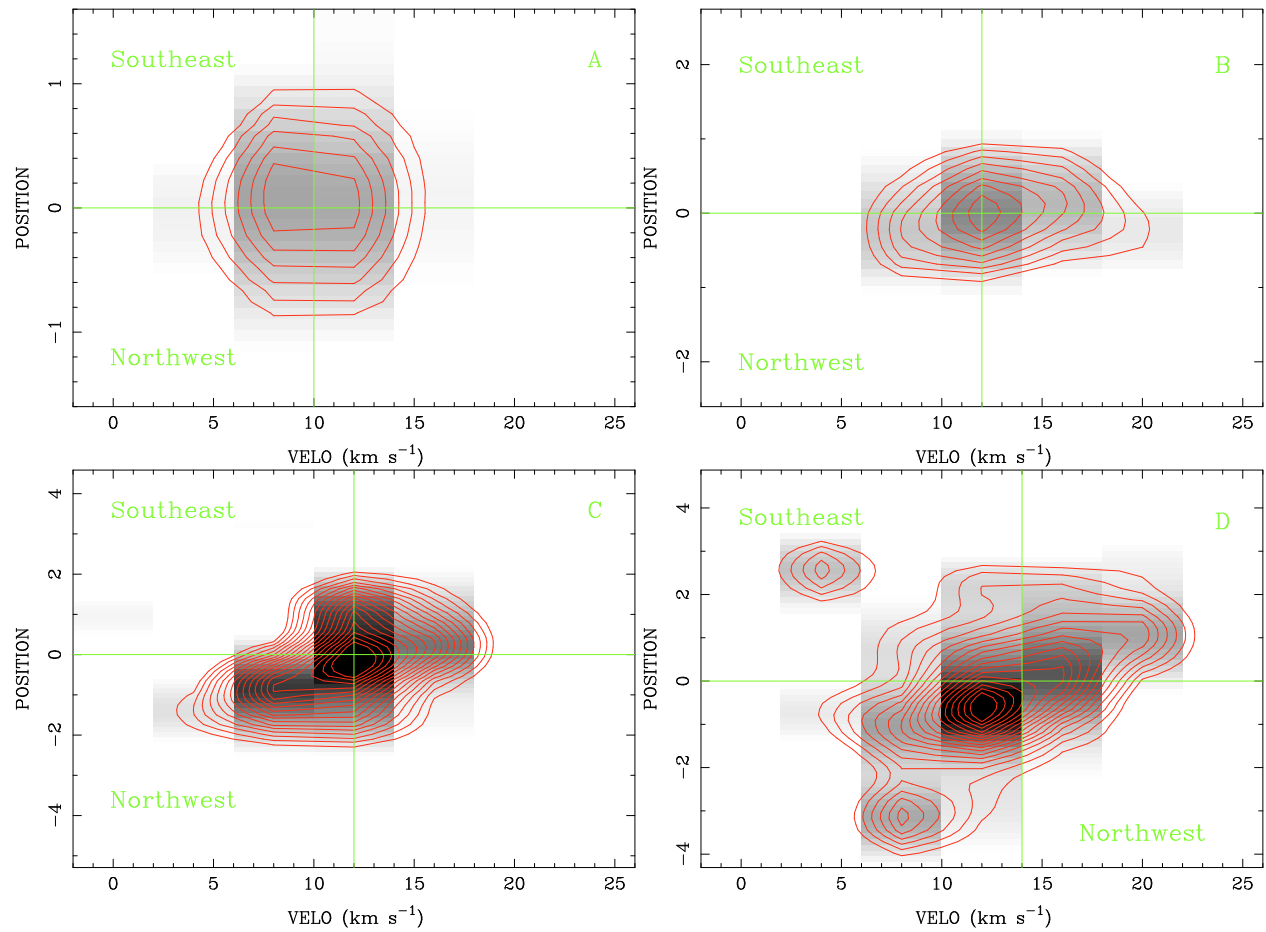

Fig. 3. Position-velocity diagrams of the four transversal cuts (along a PA $135^{\circ}$ ) across the outflow's redshifted component shown in Fig. 2 . The contours are $-3,3-20,23,25,27$ and 30 times 20 mJy beam $^{-1}$, the rms-noise of the image. The systemic LSR velocity of the ambient molecular cloud here is about 7 to $8 \mathrm{~km} \mathrm{~s}^{-1}$. The green lines in each panel mark the position of the symmetry axis of the collimated jet and the LSR radial velocities of the molecular bullet from Table 1 . The velocity resolution is $4 \mathrm{~km} \mathrm{~s}^{-1}$. The scale is in arcsec. Note that the blue- and red-shifted velocities here are given with respect to the outflow's mean velocity at a given distance from the source. 


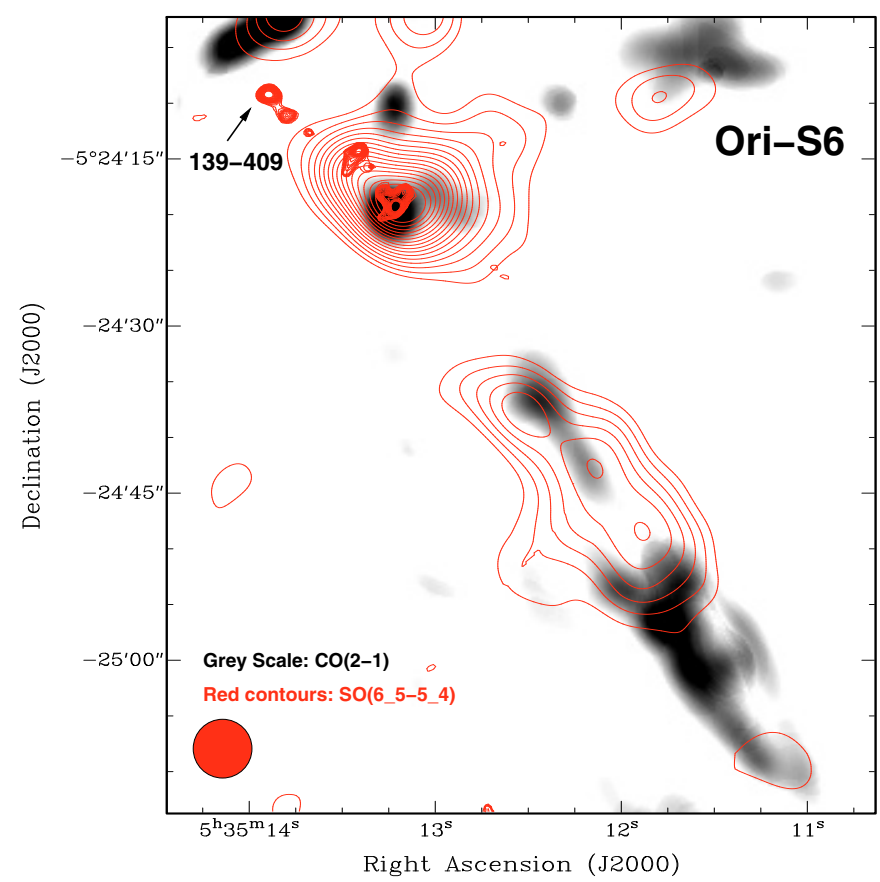

Fig. 4. SMA $\mathrm{SO}\left(6_{5}-5_{4}\right)$ low resolution integrated intensity map of the Ori-S6 molecular outflow (red thin contours) overlaid with the SMA high resolution integrated intensity of the $\mathrm{CO}(2-1)$ (grey scale) and $\mathrm{SO}\left(6_{5}-5_{4}\right)$ (red thick contours) images. Note that the $\mathrm{SO}\left(6_{5}-5_{4}\right)$ map represented in red thick contours has a better angular resolution and sensitivity than the red thin contour map, and it did not cover the entire outflow. The red thin contours start from $15 \%$ to $90 \%$ with steps of $7 \%$ the flux peak of the image, while the red thick contours start from $30 \%$ to $90 \%$ with steps of $10 \%$ the flux peak of the image. The molecular emission in the SO maps is integrated over velocities between +8 and $+25 \mathrm{~km} \mathrm{~s}^{-1}$, for the SMA CO(2-1) map from +11 to $+25 \mathrm{~km} \mathrm{~s}^{-1}$. We tapered the SMA SO low resolution data (red thin contours) to obtain a better sensitivity. The synthesized beam of the red thin contour SO map is shown in the bottom left corner of the image and has a size of 6 '. $^{\prime} 0 \times$ 5 ". 2 with a PA $=-5.5^{\circ}$.

appears to drop less steeply than its blueshifted counterpart. If the shell's rotation were radius-independent, this magnitude should be the same for both. If on the other hand the rotation speed increased outward, the "blueshifted" outer edge would have a still lower radial velocity, the "redshifted"one a higher one than in the radius-independent case, thus explaining this apparent asymmetry. With the aid of such asymmetries one might in principle be able to estimate the forces (magnetic?) that determine the rotation dynamics of the outflow shell.

\subsection{The envelope}

In Fig. 7 we show the large envelope surrounding the jet as mapped by the APEX telescope using the $\mathrm{CO}(7-6)$ and $\mathrm{CO}(6-5)$ lines.

The single-dish observations of $\mathrm{CO}(2-1),(6-5)$, and (7-6) complement the SMA picture. Of course at the velocities of the ambient gas these lines are optically very thick; it therefore takes the rare isotopomeres to discern any near-ambientvelocity structure close to the outflow axis. ${ }^{13} \mathrm{CO}(2-1)$ shows a clear and simple picture: over a distance of at least some $50^{\prime \prime}$ along the outflow two ranges of increased intensity run parallel to the outflow axis, one on either side, of constant and equal distance $\left(\leq 15^{\prime \prime}\right)$ from the axis, and of equal intensity if observed around $v_{\mathrm{LSR}}=7.7 \mathrm{~km} \mathrm{~s}^{-1}$. At lower velocities the ridge

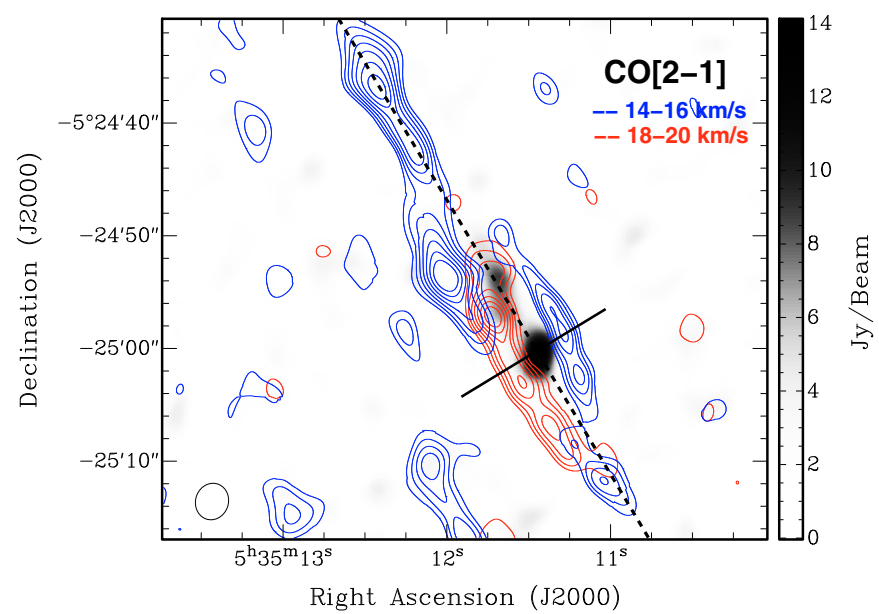

Fig. 5. SMA integrated intensity $\mathrm{CO}(2-1)$ contour map of the S6-Ori outflow showing the rotating shell (blue and red contours) and the inner high velocity jet (grey scale). The contours are from 15\% to $90 \%$ with steps of $10 \%$ of the peak of the line emission, the peak being $11 \mathrm{Jy}$ beam $^{-1}$. The redshifted emission (red contours) is integrated from 14 to $16 \mathrm{~km} \mathrm{~s}^{-1}$, the blueshifted one (blue contours) from 18 to $20 \mathrm{~km} \mathrm{~s}^{-1}$, and the grey scale from 20 to $30 \mathrm{~km} \mathrm{~s}^{-1}$. Note that the blueand red-shifted velocities refer to the outflow's mean velocity at a given distance from the source. The dashed line marks approximately the outflow's symmetry axis, and the continuous black line traces the positions where the position-velocity diagram in Fig. 6 was made. The synthesized beam is shown in the bottom left corner of the image and has a size of $3^{\prime \prime} .44 \times 22^{\prime \prime} 93$ with a PA $=-4.9^{\circ}$. Note that the outflow accelerates from top to bottom.

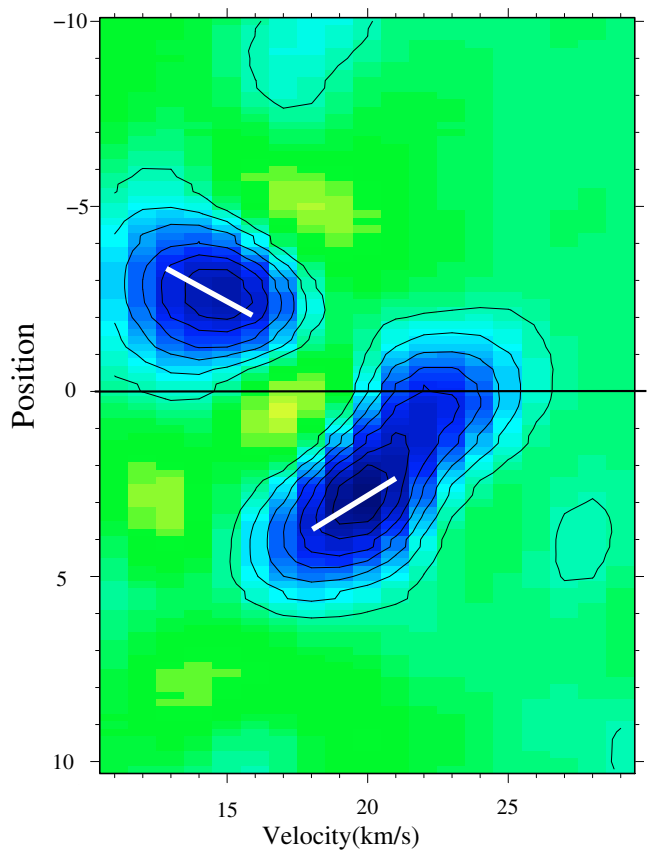

Fig. 6. Position-velocity diagram of the transversal cut (along PA $125^{\circ}$ ) marked in Fig. 5, across the outflow's redshifted bowshock. The contours are $30-90 \%$ of the peak of the line emission, the peak being $3.50 \mathrm{Jy}$ beam $^{-1}$. The black line marks the position of the symmetry axis of the molecular bowshock. The velocity and spatial resolutions are $1 \mathrm{~km} \mathrm{~s}^{-1}$ and $\sim 3^{\prime \prime}$, respectively. The spatial scale is in arcsec. For the white lines see text.

to the north-west begins to dominate, at higher values the southeastern one. We thus suspect the ambient LSR velocity in the immediate vicinity of the outflow to be around $7.7 \mathrm{~km} \mathrm{~s}^{-1}$, with 


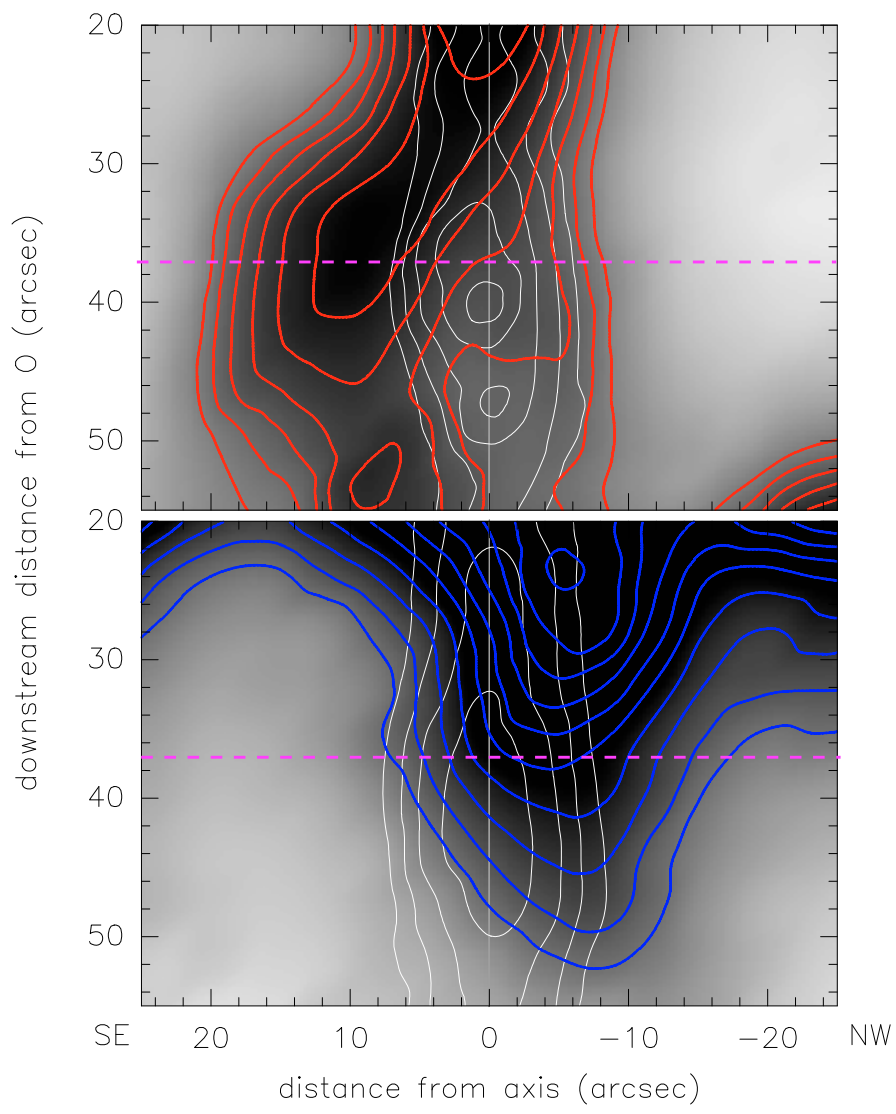

Fig. 7. CO channel maps (channel width equal to $1.5 \mathrm{~km} \mathrm{~s}^{-1}$ ) centered on velocities $12.0 \mathrm{~km} \mathrm{~s}^{-1}$ (top, $\mathrm{CO}(7-6)$ ) resp. $4.25 \mathrm{~km} \mathrm{~s}^{-1}$ (bottom, $\mathrm{CO}(6-5)$ ). The higher-velocity components of the outflow (here: integrated from 14.5 to $15.1 \mathrm{~km} \mathrm{~s}^{-1}$ ) are indicated by the white lines. Note that in the top panel a contribution from the outflow proper has been cursorily removed (see text). We estimated this outflow contribution by extrapolating from higher velocities down to the relevant velocity value. This can be done because the outflow's Gaussian cross section and amplitude at any position from the origin vary only slowly with velocity. The coordinate system here is rotated about the point $\mathrm{O}$ by $-28^{\circ}$. The pink line marks the position where the PV digrams shown in Figs. 9 and 10 were made. The red and blue contours represent the red- and blueshifted $\mathrm{CO}$ emission, respectively.

the two ridges marking the edges of a tubular wall that surrounds the flow axis. The depression between the ridges seems relatively stronger for the rarer isotopomere $\mathrm{C}^{18} \mathrm{O}$, maybe indicating preferential destruction due to less self-shielding against the UV generated in the jet's shock. A typical ${ }^{13} \mathrm{CO}$ intensity cross section through the outflow is shown in Fig. 8 for $v_{\mathrm{LSR}}=$ $7.7 \mathrm{~km} \mathrm{~s}^{-1}$, taken at a downstream distance of $45^{\prime \prime}$ from the bending point (marked by the white dot in Fig. 1). This point serve as the zero-point is from now on for all single-dish distance determinations along the outflow, because the outflow follows a straight line from here. Its coordinates are RA $=05^{\mathrm{h}} 35^{\mathrm{m}} 13.04^{\mathrm{s}}$, $\operatorname{dec}=-05^{\circ} 24^{\prime} 20.0^{\prime \prime}(\mathrm{J} 2000.0)$.

At velocities a few $\mathrm{km} \mathrm{s}^{-1}$ away from near-ambient values a structural asymmetry appears in the emission of both $\mathrm{CO}(2-1)$ and ${ }^{13} \mathrm{CO}(2-1)$, see Fig. 8. Some 3 to $4 \mathrm{~km} \mathrm{~s}^{-1}$ below the $7.7 \mathrm{~km} \mathrm{~s}^{-1}$ value the intensity of both species is strongest along a strip situated between the ambient tubular wall and the outflow axis (as defined by the high-velocity cross-section profile) on the north-western side of the axis, at $v_{\text {LSR }}$ some 3 to $4 \mathrm{~km} \mathrm{~s}^{-1}$ above this value the emission peaks along a corresponding strip on the south-eastern side. These two zones are spatially

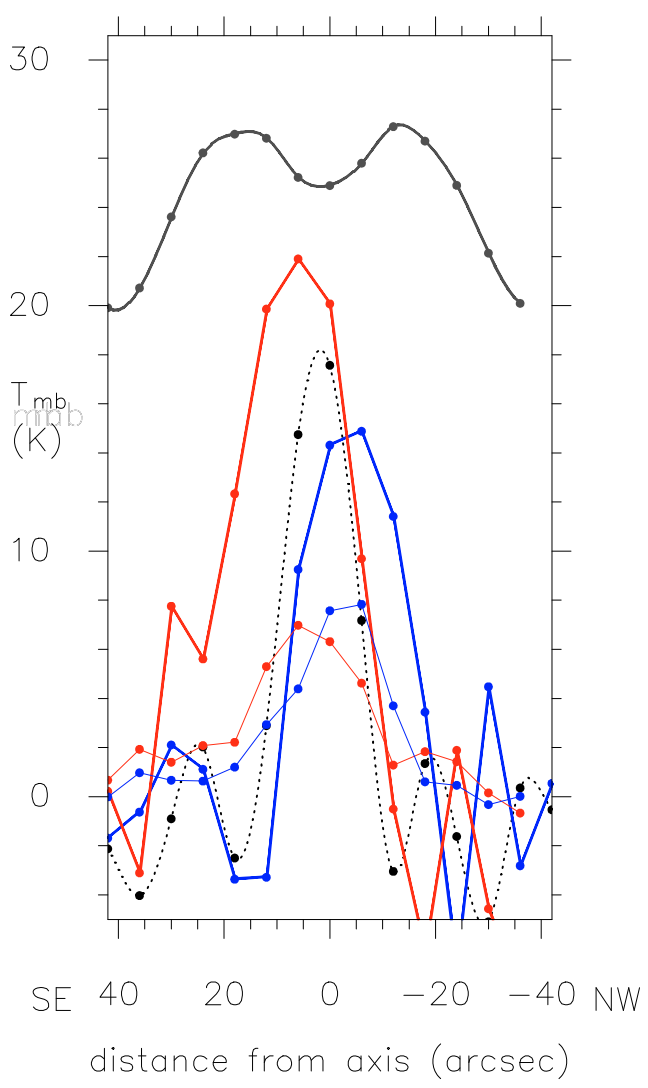

Fig. 8. Variation of line intensities (IRAM $30 \mathrm{~m}$ ) across the outflow at a downstream distance of $45^{\prime \prime}$ from point $\mathrm{O}$ (see Fig. 1): the black line is ${ }^{13} \mathrm{CO}(2-1)$ at velocities around $7.7 \mathrm{~km} \mathrm{~s}^{-1}$ (i.e. ambient), shifted upwards by $8 \mathrm{~K}$. The dotted black line is also ${ }^{13} \mathrm{CO}(2-1)$ at velocities around $15 \mathrm{~km} \mathrm{~s}^{-1}$. The blue and red lines show the spatial separation between redshifted $\left(11.6 \mathrm{~km} \mathrm{~s}^{-1}\right)$ and blueshifted $\left(4.6 \mathrm{~km} \mathrm{~s}^{-1}\right)$ components of the Ori-S6 outflow from $\mathrm{CO}(2-1)$ (strong lines) and ${ }^{13} \mathrm{CO}(2-1) \times$ 7 (fainter lines).

separated by $\sim 10^{\prime \prime}$ to $15^{\prime \prime}$ and by $\sim 7 \mathrm{~km} \mathrm{~s}^{-1}$ in radial velocity, and this asymmetry extends downstream to projected distances of at least $2.5 \times 10^{4} \mathrm{AU}$ from the source. The sense of the asymmetry corresponds to our SMA results, but the velocities in question have a different context: the SMA variations are a superposition of an asymmetry of a few $\mathrm{km} \mathrm{s}^{-1}$ onto the high-velocity components of the outflow, the single-dish data concern motions outside the core flow zone.

Typical $p-v$ diagrams taken perpendicularly across the outflow at a distance of $37^{\prime \prime}$ from point $\mathrm{O}$, as obtained with the somewhat higher resolutions of the APEX measurements compared with those of IRAM CO(2-1), are shown in Fig. 9. No important long-range gradient of the ambient velocities is evident, and there appears in particular no obvious tilt across the outflow axis (the horizontal white line). However, in both diagrams one notes conspicuous excursions of the iso-intensity lines, somewhat anti-symmetrically offset from the outflow's axis, at velocities slightly beyond the optically thick $v_{\text {LSR }}$ values, i.e. below 5 resp. above $11 \mathrm{~km} \mathrm{~s}^{-1}$ (big white circles). These might seem spurious; but when observing at different downstream positions one notes their appearance in each $p-v$ crosscut between $20^{\prime \prime}$ and $60^{\prime \prime}$ from point $\mathrm{O}$ (see Fig. 10), contrary to some other local excursions also visible in Fig. 9, which do not persist across this range. Figure 10 does show noticeable differences between the high- and the low-velocity diagrams. But complete antisymmetry between the encircled regions of Fig. 9 cannot be expected 


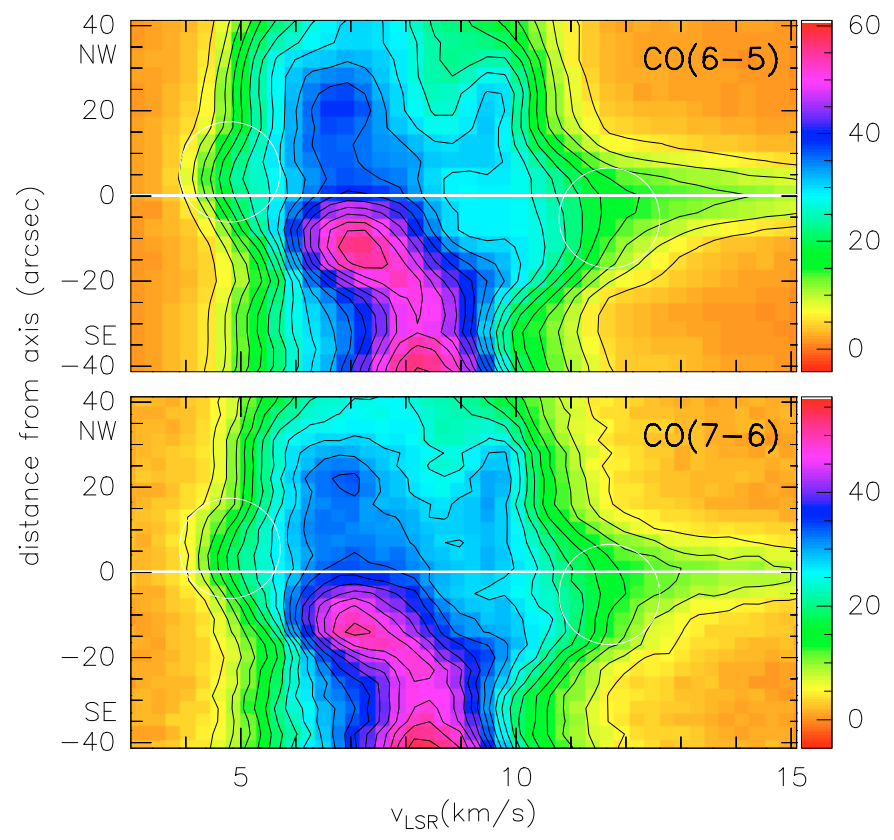

Fig. 9. Position-velocity diagrams $(\mathrm{CO}(6-5)$ and $\mathrm{CO}(7-6))$ of the perpendicular crosscut through the outflow at a downstream distance 37" from point $\mathrm{O}$ (see Fig. 8). The outflow axis is marked by the horizontal white line, the regions discussed in the text for velocity asymmetry is indicated by the circles. Colour scale is $T_{\mathrm{A}}^{*}$ in $\mathrm{K}$.

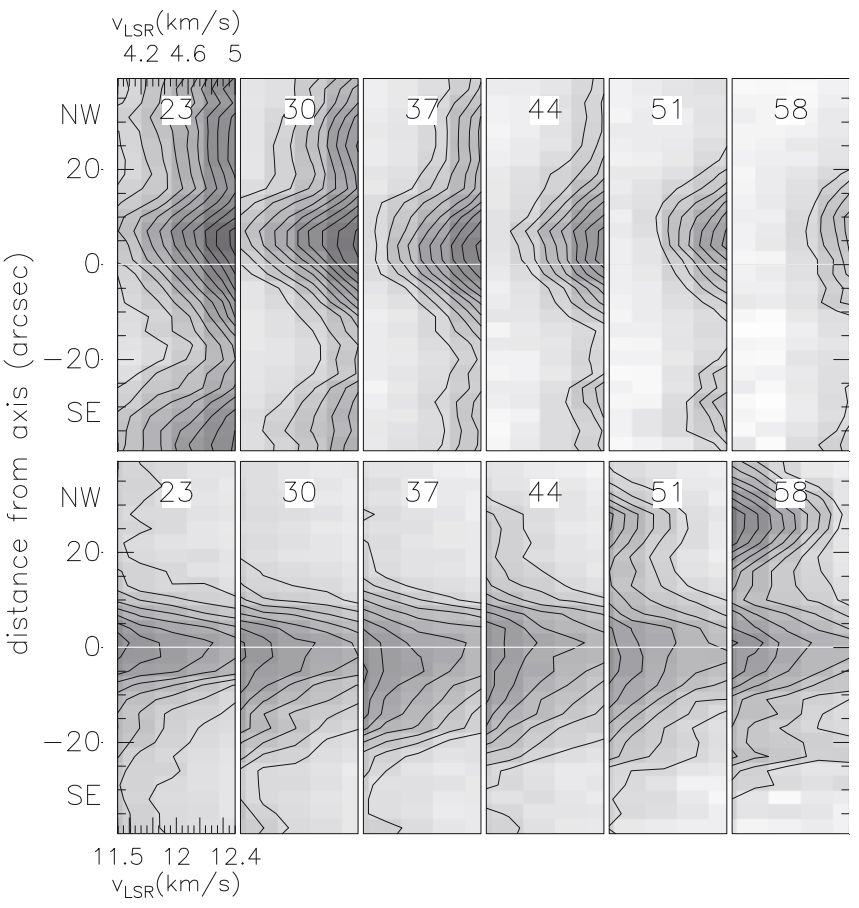

Fig. 10. Sections of the position-velocity diagrams $(\mathrm{CO}(7-6))$ of perpendicular crosscuts through the outflow at different distances (given by the numbers on the panels, arcsec) from point $\mathrm{O}$. The outflow axis is marked by the horizontal grey line. Top: blueshifted velocities between 4 and $5 \mathrm{~km} \mathrm{~s}^{-1}$, bottom: redshifted ones from 11.5 to $12.5 \mathrm{~km} \mathrm{~s}^{-1}$. Note that the structures near the outflow axis persist for at least six beams.

because the outflow proper will have different effects on the two velocity windows, contaminating the redshifted rotation component. This component seems to extend further from the axis, and it may consist of two somewhat distinct parts, one at the

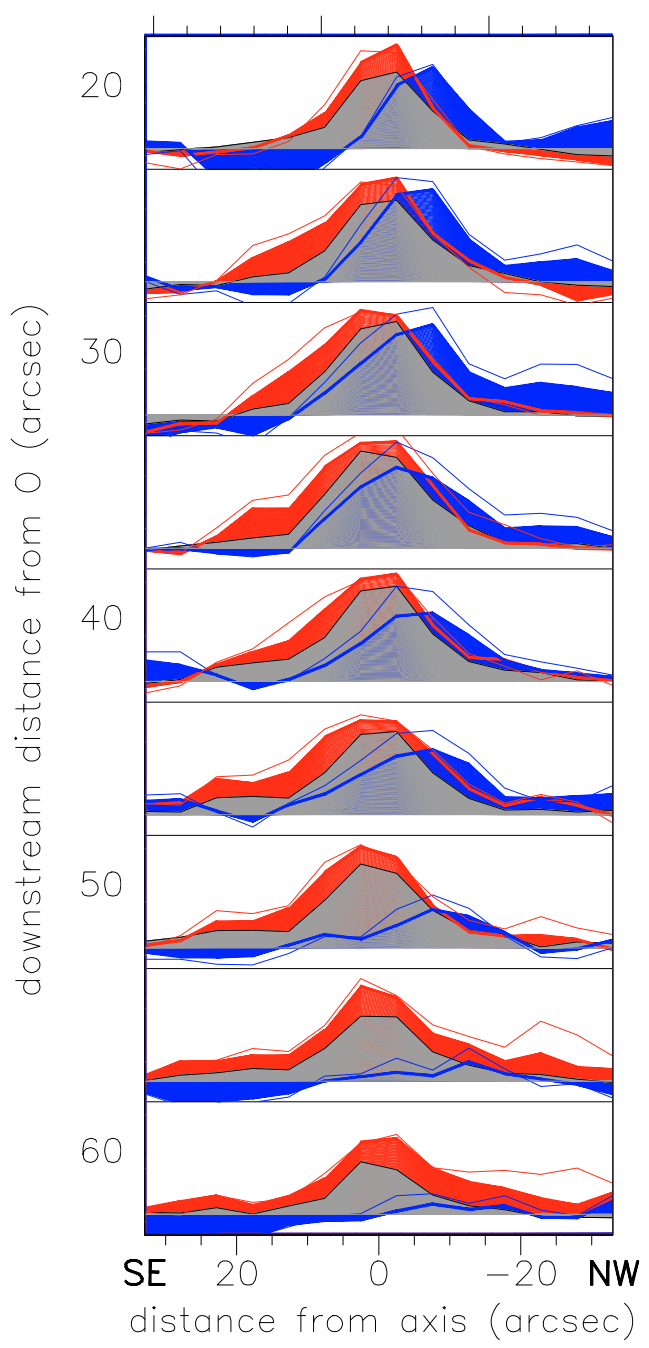

Fig. 11. Red-blue asymmetry of $\mathrm{CO}(6-5)$ emission across the outflow at increasing distances from point $\mathrm{O}$. Grey: Cross section at $15 \mathrm{~km} \mathrm{~s}^{-1}$ (core of the outflow), red: at $12.7 \mathrm{~km} \mathrm{~s}^{-1}$, blue: at $4.5 \mathrm{~km} \mathrm{~s}^{-1}$, each integrated over a velocity interval of a width of $0.5 \mathrm{~km} \mathrm{~s}^{-1}$. The $T_{\mathrm{mb}}$ scale is -4 to $22 \mathrm{~K}$. The thin colored lines show the distributions by $0.5 \mathrm{~km} \mathrm{~s}^{-1}$ closer to the ambient velocity.

axial distance of the blueshifted component (about $5^{\prime \prime}$ ), the other one father away (see panel 37 of Fig. 10). Higher resolution studies and better knowledge of the central outflow's contribution are needed to decide this issue.

In the two transition regions between the typical excursion and the ambient velocities, i.e. at around 6 resp. $10.5 \mathrm{~km} \mathrm{~s}^{-1}$, there are hints in Fig. 11 (as well as at other downstream distances) of emission deficits at about the same offsets from the axis as those where the excursion emission peaks (Iso-intensity lines change from convex to concave along the $v_{\text {LSR }}$ axis). This seems to indicate in situ acceleration in opposite directions on either side of the outflow, rather than a mere ambient velocity gradient to be at the root of the observed velocity antisymmetry.

Spatial intensity profiles across the flow (Fig. 11), taken for the two velocity excursions in question at various downstream distances from point O, clearly show a persistent "red-blue" asymmetry about the axis that is defined by the high-velocity crosscut here depicted in grey. Mapping the two "excursion channels" of 3.5 to $5.0 \mathrm{~km} \mathrm{~s}^{-1}$ resp. 11.25 to $12.75 \mathrm{~km} \mathrm{~s}^{-1}$ results in the spatial distributions of Fig. 7 which represent the 
SMA CO(2-1) line emission

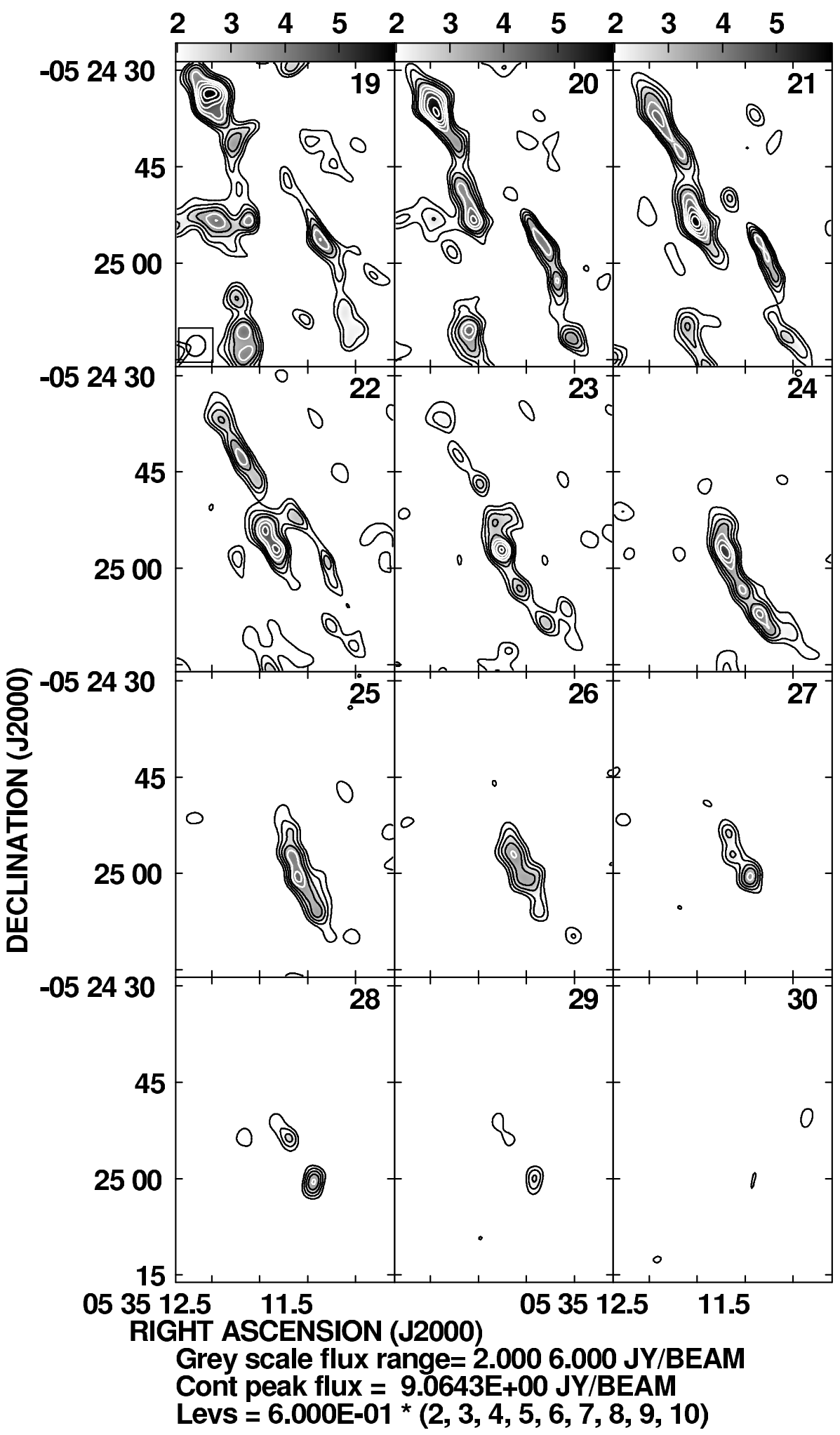

Fig. 12. SMA channel maps of the $\mathrm{CO}(2-1)$ line emission from the outflow Ori-S6. The emission is summed in velocity bins of $1 \mathrm{~km} \mathrm{~s}^{-1}$. The central velocity is indicated in the upper right-hand corner of each panel (the systemic velocity of the ambient molecular cloud is about $6-7 \mathrm{~km} \mathrm{~s}^{-1}$ ). considerable velocity difference of some 6 to $8 \mathrm{~km} \mathrm{~s}^{-1}$ over a distance of around 10-15" or 4-6 $410^{3}$ AU. Of course this variation does not signal an actual velocity difference of $6 \mathrm{~km} \mathrm{~s}^{-1}$ or so between the two sides, the bulk of the line profiles being hidden by a large optical depth in a way that only their extreme wings can be seen. The fact that either side predominantly shows just one of the two wings, the "red" or the "blue" one, indicates however that the true radial velocity shift across the flow must be roughly on the order of at least $0.5 \mathrm{~km} \mathrm{~s}^{-1}$, corresponding to $20 \mathrm{~km} \mathrm{~s}^{-1}$ per pc; were it much smaller, then one would measure about the same wing emission on either side. This in turn indicates that the rotation velocity between the inner SMA region and the annular zone of axial distance $R=10^{\prime \prime}$ to $15^{\prime \prime}$ does not decrease much faster than $1 / R$. 
SMA SO(6,5-5,4) line emission

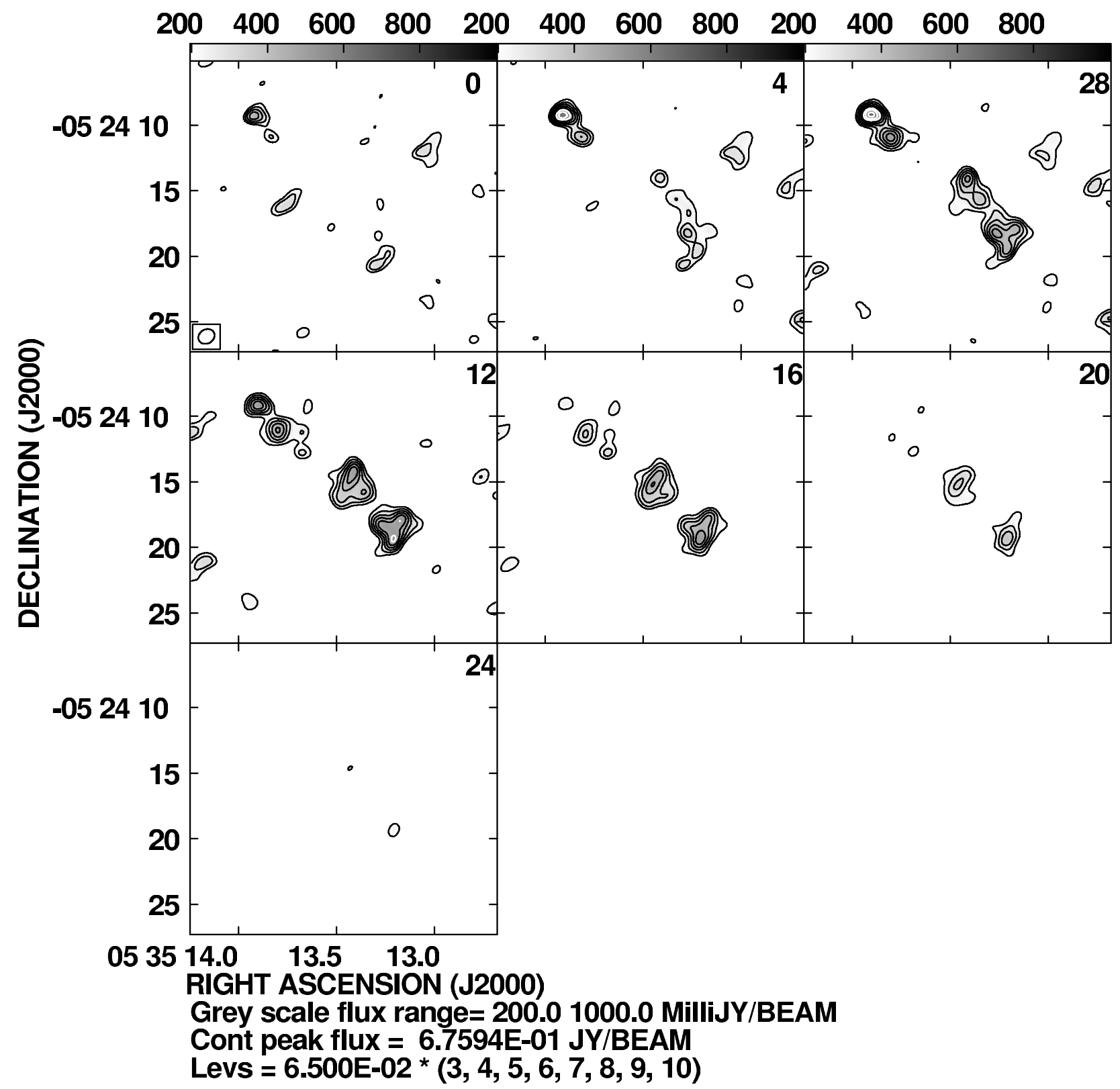

Fig. 13. SMA channel maps of the $\mathrm{SO}\left(6_{5}-5_{4}\right)$ line emission from the outflow Ori-S6. The emission is summed in velocity bins of $4 \mathrm{~km} \mathrm{~s}^{-1}$. The central velocity is indicated in the upper right-hand corner of each panel (the systemic velocity of the ambient molecular cloud is about $6-7 \mathrm{~km} \mathrm{~s}^{-1}$ ).

Note that in order to somewhat correct Fig. 7 for the contribution from the outflow proper to the redshifted intensities we have rather cursorily subtracted part of the outflow's higher velocities (between 14 and $18 \mathrm{~km} \mathrm{~s}^{-1}$ ) from the data.

\section{Discussion}

Three independent observations all show velocity asymmetries about the outflow axis which suggest rotation on different length scales. Seen along the redshifted lobe down from the origin this rotation would be clockwise for the SO clumps, the CO jet shell, and the ambient envelope alike.

Although this congruence of velocity shifts on three different length scales lends some credence to a rotation model for Ori-S6, we have to discuss alternative explanations for the observed redblue asymmetries. The most obvious alternative would be a general large-scale velocity gradient in the region. Indeed, as the $p-v$ diagram of Fig. 9 shows, there is some overall velocity change in the brightest component, from higher to lower velocities along a direction roughly SE to NW. Between the edges of this diagram, i.e. over a distance of $80^{\prime \prime}$, the peak $v_{\mathrm{LSR}}$ is seen to change by about $1 \mathrm{~km} \mathrm{~s}^{-1}$. Were this a smooth gradient, it would amount to $\sim 5 \mathrm{~km} \mathrm{~s}^{-1}$ per pc, a value high but not uncommon for molecular cloud clumps. Over the $5^{\prime \prime}$ resp. 10" distances between the two sides of the CO jet shell (Fig. 5) resp. the "ambient" CO tube (Fig. 11) this would however amount to velocity asymmetries below $0.1 \mathrm{~km} \mathrm{~s}^{-1}$, much less than what is observed. In fact, over these distances the brightest component does not seem to vary at all in velocity; only beyond, and then 
only in the SE part of the diagram over the very limited extent of some $10^{\prime \prime}$, a much larger gradient appears, on the order of $60 \mathrm{~km} \mathrm{~s}^{-1}$ per parsec. This huge gradient connects suspiciously to the velocity dominant still further away from the flow. One could postulate such a large, spatially very limited velocity gradient that by chance coincides with the outflow positions. But that gradient would have to be closely matched with the flow for these asymmetries to be evident over a length of scales on the order of $60^{\prime \prime}$. This seems unlikely. Also, the convex-concave transitions mentioned above appear to favor in situ acceleration over any effects of an ambient velocity gradient. We thus discard the notion of the asymmetries being caused by such a gradient in the ambient gas.

Since the outflow originates in a binary system (Zapata et al. 2007) a recent calculation (Murphy et al. 2008) of two nearly parallel jets of unequal speed stemming from such a system may be of relevance for Ori-S6. In that model the two jets eventually merge, as witnessed by a persistent kink in the final structure. Furthermore, due to binarity precession begins to show after some time in the form of a bending jet trajectory. Although neither of these effects can as yet be definitely excluded for our inner jet, SMA data of the large-distance CO structure clearly speaks against any sizeable precession or kink. Nor would it seem likely that the two flow velocities should not over gradually adjust to each other their large common path and thus wipe out any initial differences in speed. We therefore look for other explanations for the observed velocity jumps across the flow axis.

Soker (2005) alternative proposal that left-right asymmetries in jets could result from (non-magnetic) interaction at the base between jet and a warped disk, would on the other hand at the very least in our case require a warp spatially static over the long times that it takes to build up the outflow out to $100^{\prime \prime}$ from the source: $0.2 \mathrm{pc} / 15 \mathrm{~km} \mathrm{~s}^{-1}$ is of order 13000 years, so even accounting for possibly reducing projection factors a very large period of standstill would be demanded of the close binary system at the source of Ori-S6. Hence we also discard Soker's mechanism and relate the observed jumps to rotation instead.

Where would this rotation originate? The $\mathrm{CO}$ jet shown in Fig. 5 is considered to be ejected from a rotating protostellar disk and then accelerated and collimated by MHD forces. Anderson et al. (2003) provide a formula that allows the relation of jet properties measured at large distances from the disk to the position (the "footpoint") on the disk from where the observed jet section first emerges:

$$
\begin{aligned}
\varpi_{0} \approx & 0.7 \mathrm{AU}\left(\frac{\varpi_{\infty}}{10 \mathrm{AU}}\right)^{2 / 3}\left(\frac{v_{\phi, \infty}}{10 \mathrm{~km} \mathrm{~s}^{-1}}\right)^{2 / 3} \\
& \times\left(\frac{v_{p, \infty}}{100 \mathrm{~km} \mathrm{~s}^{-1}}\right)^{-4 / 3}\left(\frac{M_{*}}{1 M_{\odot}}\right)^{1 / 3} .
\end{aligned}
$$

Here $\varpi_{\infty}$ is the observed radial distance of the jet shell from the flow axis, $\varpi_{0}$ the radius on the disk from where that shell's material leaves, $v_{\phi, \infty}$ and $v_{p, \infty}$ are the toroidal and poloidal velocities observed for the shell at $\varpi_{\infty}$, and $M_{*}$ the mass of the (proto)star at the center of the disk. In our case we assume from observation $\varpi_{\infty} \sim 2.5^{\prime \prime}=1000 \mathrm{AU}, v_{\phi, \infty} \sim 2 \mathrm{~km} \mathrm{~s}^{-1}, v_{p, \infty} \sim 10 / \sin \alpha \mathrm{km} \mathrm{s}^{-1}$ and $M_{*} \sim 2$ to $5 M_{\odot}$, with $\alpha$ the unknown angle between flow direction and the plane of the sky. This result in a footpoint radius of $140 /(\sin \alpha)^{4 / 3}$ for the jet component shown in Fig. 5. Since the apparent poloidal flow velocity of $\sim 10 \mathrm{~km} \mathrm{~s}^{-1}$ relative to ambient is relatively low for molecular jets it seems likely that $\alpha$ is small. For $\alpha \leq 30^{\circ}$ the footpoint radius would drop to below $56 \mathrm{AU}$, a value that fits the above-mentioned disk dimensions of the binary components very well. Furthermore, similar values for the footpoint radius are found for the $\mathrm{SO}\left(6_{5}-5_{4}\right)$ molecular jet.

A similar estimate for the $\mathrm{CO}$ envelope of nearly ambient velocity, shown in cross-section in Fig. 7, must await more detailed investigations since its apparent $v_{p, \infty}$ value is (very) small and not determined; likewise $v_{\phi, \infty}$ can be estimated only very roughly, as explained above - the asymmetric wings say little quantitatively about the bulk toroidal motions of the envelope. A trial with $\varpi_{\infty} \sim 5^{\prime \prime}, v_{\phi, \infty} \sim 0.4 \mathrm{~km} \mathrm{~s}^{-1}$, and $v_{p, \infty} \sim 1 \mathrm{~km} \mathrm{~s}^{-1}$ would result in footpoint radii about a factor of ten larger than the CO jets shell's, suggesting an origin quite different from that of the jet's disk.

Anderson et al. (2003) also provide an expression for the ratio between toroidal and poloidal components of the magnetic field strength at the observed jet positions (see their Eq. (2)). Using the same parameter values as employed for estimating the jet shell's footpoint radius and setting $\alpha$ to $30^{\circ}$ we arrive at a field strength ratio (toroidal over poloidal) of around six. The magnetic field in the shell thus seems to be tightly wound up, thereby keeping the shell material well collimated by its hoop stresses.

Where in the system 139-409 the jet actually originates has yet to be determined. The sense of rotation of the circumbinary ring is nearly opposite to that of the jet and the outflow, and the jet leaves the system under an angle of $45^{\circ}$ with the ring plane. One should therefore expect the origin of the flow to be at the circumstellar disk of one of the binary components. The two disks need not align with the ring. Further deep observations of highest resolution will be required to clarify the jet-disk connection.

\section{Summary}

The Ori-S6 outflow presents a promising laboratory for future studies of magneto-centrifugal models of jet acceleration. We have observed the compact jet and its larger-scale molecular envelope on three different spatial scales and found the following:

- the SO bullets in the jet, its CO shell and also the more distant $\mathrm{CO}$ envelope all show rotation about the outflow axis; the sense of rotation is the same for all;

- the inner jet is composed of individual bullets that appear to follow a solid body rotation law with peak $v_{\text {rot }}$ values around $5 \mathrm{~km} \mathrm{~s}^{-1}$. The CO shell, at a distance of about $\sim 1000 \mathrm{AU}$ from the axis, rotates at $v_{\text {rot }} \sim 2 \mathrm{~km} \mathrm{~s}^{-1}$; the $\mathrm{CO}$ envelope, at a distance of $\geq 2000 \mathrm{AU}$ from the axis, i.e. several times the bullets' radii, rotates at $v_{\text {rot }} \geq 0.5 \mathrm{~km} \mathrm{~s}^{-1}$;

- the rotation is observed out to at least $25000 \mathrm{AU}$ downstream from the source;

- the magnetic field lines embedded in the jet's CO shell can well thread a protostellar disk of radius $\sim 50$ AU. For the wider $\mathrm{CO}$ envelope the footpoint in the equatorial plane of the disk must lie considerably further out;

- the exact identification of the outflow source remains open since at the obvious position there is a circumbinary ring containing two intermediate mass stars. Orientation and sense of rotation of the ring do not coincide with those of the outflow.

All these topics are amenable to more extended observations that aim at problems of star formation, such as angular momentum budgets and high-velocity mass ejection.

Acknowledgements. We would like to thank Dr. Bernd Klein for having taken the recent CO data on the $30 \mathrm{~m}$ telescope, and Dr. Rainer Mauersberger for reactivating the IRAM data of 1989. Facilities: IRAM $30 \mathrm{~m}$ APEX and SMA. 


\section{References}

Anderson, J. M., Li, Z.-Y., Krasnopolsky, R., \& Blandford, R. D. 2003, ApJ, 590, L107

Bacciotti, F., Ray, T. P., Mundt, R., Eislöffel, J., \& Solf, J. 2002, ApJ, 576, 222

Cerqueira, A. H., Velázquez, P. F., Raga, A. C., Vasconcelos, M. J., \& de Colle F. 2006, A\&A, 448, 231

Chrysostomou, A., Bacciotti, F., Nisni, B., et al. 2008, A\&A, 482, 575

Codella, C., Cabrit, S., Gueth, F., et al. 2007, A\&A, 462, L53

Coffey, D., Bacciotti, F., Woitas, J., Ray, T. P., \& Eislöffel, J. 2004, ApJ, 604, 758

Coffey, D., Bacciotti, F., Ray, T. P., Eislöffel, J., \& Woitas, J. 2007, ApJ, 663, 350

Davis, C. J., Berndsen, A., Smith, M. D., Chrysostomou, A., \& Hobson, J. 2000, MNRAS, 314, 241

Henney, W. J., O’Dell, C. R., Zapata, L. A., et al. 2007, AJ, 133, 2192

Ho, P. T. P., Moran, J. M., \& Lo, K. Y. 2004, ApJ, 616, L1

Königl, A., \& Pudritz, R. E. 2000, Protostars and Planets IV, 759

Launhardt, R., Pavlyuchenkov, Y., Gueth, F., et al. 2009, A\&A, 494, 147

Lee, C.-F., Ho, P. T. P., Bourke, T. L., et al. 2008, ApJ, 685, 1026

Menten, K. M., Reid, M. J., Forbrich, J., \& Brunthaler, A. 2007, A\&A, 474, 515

Mezger, P. G., Zylka, R., \& Wink, J. E. 1990, A\&A, 228, 95

Muders, D., \& Schmid-Burgk, J. 1992, in Astronomische Gesellschaft Abstract Series, 45
Mundy, L. G., Scoville, N. Z., Baath, L. B., Masson, C. R., \& Woody, D. P. 1986, ApJ, 304, L51

Murphy, G. C., Lery, T., O’Sullivan, S., et al. 2008, A\&A, 478, 453

O'Dell, C. R., Muench, A., Smith, N., \& Zapata, L. 2008, Star Formation in the Orion Nebula II: Gas, Dust, Proplyds and Outflows, ed. B. Reipurth, 544

Pety, J., Gueth, F., Guilloteau, S., \& Dutrey, A. 2006, A\&A, 458, 841

Pudritz, R. E., Ouyed, R., Fendt, C., \& Brandenburg, A. 2007, in Protostars and Planets V, ed. B. Reipurth, D. Jewitt, \& K. Keil, 277

Schmid-Burgk, J., Guesten, R., Mauersberger, R., Schulz, A., \& Wilson, T. L. 1990, ApJ, 362, L25

Scoville, N. Z., Carlstrom, J. E., Chandler, C. J., et al. 1993, PASP, 105, 1482

Shang, H., Li, Z.-Y., \& Hirano, N. 2007, in Protostars and Planets V, ed. B. Reipurth, D. Jewitt, \& K. Keil, 261

Shu, F. H., Najita, J. R., Shang, H., \& Li, Z.-Y. 2000, Protostars and Planets IV, 789

Soker, N. 2005, A\&A, 435, 125

Woitas, J., Bacciotti, F., Ray, T. P., et al. 2005, A\&A, 432, 149

Zapata, L. A., Rodríguez, L. F., Kurtz, S. E., \& O’Dell, C. R. 2004a, AJ, 127, 2252

Zapata, L. A., Rodríguez, L. F., Kurtz, S. E., O’Dell, C. R., \& Ho, P. T. P. 2004b, ApJ, 610, L121

Zapata, L. A., Rodríguez, L. F., Ho, P. T. P., et al. 2005, ApJ, 630, L85

Zapata, L. A., Ho, P. T. P., Rodríguez, L. F., et al. 2006, ApJ, 653, 398

Zapata, L. A., Ho, P. T. P., Rodríguez, L. F., et al. 2007, A\&A, 471, L59 